\title{
Deep convolutional network for animal sound classification and source attribution using dual audio recordings
}

\author{
Tuomas Oikarinen, ${ }^{1}$ Karthik Srinivasan, ${ }^{1}$ Olivia Meisner, ${ }^{1}$ Julia B. Hyman, ${ }^{1}$ \\ Shivangi Parmar, ${ }^{1}$ Adrian Fanucci-Kiss, ${ }^{1}$ Robert Desimone, ${ }^{1}$ Rogier Landman, ${ }^{2, a)}$ \\ and Guoping Feng ${ }^{1, b)}$ \\ ${ }^{1}$ McGovern Institute for Brain Research, Massachusetts Institute of Technology, 43 Vassar Street, Cambridge, \\ Massachusetts 02139, USA \\ ${ }^{2}$ Stanley Center, Broad Institute, 57 Ames Street, Cambridge, Massachusetts 02139, USA
}

(Received 4 October 2018; revised 28 December 2018; accepted 2 January 2019; published online 4 February 2019)

\begin{abstract}
This paper introduces an end-to-end feedforward convolutional neural network that is able to reliably classify the source and type of animal calls in a noisy environment using two streams of audio data after being trained on a dataset of modest size and imperfect labels. The data consists of audio recordings from captive marmoset monkeys housed in pairs, with several other cages nearby. The network in this paper can classify both the call type and which animal made it with a single pass through a single network using raw spectrogram images as input. The network vastly increases data analysis capacity for researchers interested in studying marmoset vocalizations, and allows data collection in the home cage, in group housed animals. (C) 2019 Acoustical Society of America. https://doi.org/10.1121/1.5087827
\end{abstract}

$[\mathrm{PG}]$

Pages: 654-662

\section{INTRODUCTION}

Convolutional neural networks (in two-dimensions) have seen a lot of success in the fields of environmental and animal sound classification (Boddapati et al., 2017). Using convolution in both the time and frequency domain makes sense for these tasks since animal calls and environmental sounds often have distinct structure that can be clearly seen in spectrograms or other image representations of audio data. Here we present a neural network for auto detection, classification, and attribution of vocalizations in the common marmoset (Callithrix Jacchus).

The impetus for this work is research using marmosets as a primate model to study mental disorders affecting social behavior, such as autism (Jennings et al., 2016; Miller et al., 2016). In marmosets, vocal exchanges are an essential part of social interaction (Eliades and Miller, 2017), and are at least partly learned from parents and peers. Analysis of vocalizations can yield measures of vocal development, and vocal interactions can potentially be used to track sociability and learning of social rules. However, labeling vocalizations in audio recordings is labor-intensive; therefore, automation is valued highly.

Our dataset consists of dual channel recordings from normal, captive marmoset monkeys housed in pairs, where each animal wears a voice recorder. The data is annotated by researchers for a variety of call types. We present a neural network that can detect and classify the calls from each animal with high accuracy based on the spectrogram. We use one network with two convolutional streams, which are concatenated in the end and followed by a single fully

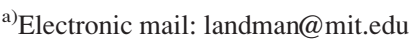

b) Also at: Stanley Center, Broad Institute, 57 Ames Street, Cambridge, MA 02139, USA.
}

connected layer with dropout before a final softmax layer that classifies both the type and the source of the animal call.

In this paper, Sec. II covers background and previous models, Sec. III describes the details of the experiment, the dataset, and the neural network model; in Sec. IV we report the results, and in Sec. V, we present a discussion and conclusions.

\section{BACKGROUND AND RELATED WORK}

The common marmoset (Callithrix Jacchus) is among the smallest primates and is gaining interest as a non-human primate model for neuroscience research (Jennings et al., 2016). The species lends itself well for studying social behavior, since marmosets have features in common with humans that are not found in every primate species, such as vocal interaction, imitation, and cooperative breeding (Miller et al., 2016). In the vocal domain, marmosets have a repertoire of at least eight call types, which occur in different conditions, and are thought to convey different information to others. For example, there are calls that serve to maintain contact with members of their group, calls that broadcast the presence of a threat and calls that signal inter-group threats (Bezerra and Souto, 2008; Miller et al., 2010). In both humans and marmosets, vocal interactions are structured and organized according to set principles (Sacks et al., 1974). The exchange of contact calls between marmosets shows a turn-taking dynamic that is comparable to turn-taking in human conversation and other interactions (Henry et al., 2015; Levinson and Torreira, 2015).

We distinguish three functions that an automatic vocalization-detection system should perform in order to vastly speed up the study of vocal interactions: Detection (whether there is a vocalization), Classification (which type of vocalization it is), and Attribution (which animal vocalized). Automated classification has been done in various species, including rodents (Kobayasi and Riquimaroux, 2012; 
Soltis et al., 2012), frogs (Pettitt et al., 2012), birds (Giret et al., 2011), bats (Prat et al., 2016) and primates (Fuller, 2014; Hedwig et al., 2014), including marmosets (Agamaite et al., 2015; Turesson et al., 2016; Zhang et al., 2018). In previous marmoset studies, it was possible to detect the vocalizations by amplitude thresholding of the band- or high-passed audio signal. Attribution was not part of these efforts. As input for classification Agamaite et al. (2015) extracted 18 acoustic features, chosen by the investigators, in both the time and frequency domain (see Table I in Agamaite et al., 2015). Turesson et al. (2016) used Linear Predictive Coding filters for feature extraction. Zhang et al. (2018) is the most recent work to automatically classify marmoset calls with higher classification accuracy and low frame error rate. They employed a deep recurrent neural network with fully connected layers and Long Short Term Memory (LSTM) (Graves et al., 2013), in some cases. The data acquisition was from only one animal at a time and thus there is no source attribution or the need to separate background calls. Furthermore, in Zhang et al. (2018), the audio recordings were preprocessed using bandpass filters and log-mel filter banks to manually select features to train the network. Call detection and classification were two separate processes and their performance too was evaluated individually.

The current study is different from previous marmoset studies in several ways. First, recordings were done in a noisy environment. The animals were in their home cage with their cage partner in a room with multiple other cages with animals. While there is benefit in studying animals in conditions where they feel at home and can freely interact with their cage mates, the added noise from animals, cages, air-vents, human personnel, etc., makes it that amplitude thresholding is not sufficient for detecting whether there is a vocalization. Second, our goal was to attribute vocalizations to individuals that are freely moving within the same cage. Attribution is not trivial under these circumstances, because the animals are not spatially separated. We choose to use wearable voice recorders for that reason, and attribute calls using the neural network. The ideal result would be a system where we input two raw audio files and the output is a list of one row per call and columns with start time, stop time, call type, and ID (animal 1 or animal 2).

\section{EXPERIMENT}

Our dataset consists of audio recordings done on pairs of animals sharing a cage, each wearing a voice recorder.

TABLE I. The eight call types we distinguish and the "noise" category, containing vocalizations from animals in the background, and sounds that are not from animals. Right column: the number of segments included.

\begin{tabular}{lc}
\hline \hline Call type & Number of segments \\
\hline Trill & 23549 \\
Twitter & 10614 \\
Phee & 10121 \\
Chirp & 5917 \\
Ek & 3265 \\
Trillphee & 2980 \\
Tsik & 2753 \\
Chatter & 1399 \\
Noise (no call type) & 126038 \\
\hline \hline
\end{tabular}

Between 8 and 20 other animals are present in the room, in other cages. The voice recorders (Polend mini 8GB voice recorder) are mounted at the chest in custom made jackets. The animals are thoroughly habituated to wearing the jackets before recording starts. All animal procedures are overseen by veterinary staff of the Massachusetts Institute of Technology (MIT) and Broad Institute Department of Comparative Medicine, in compliance with the National Institutes of Health Guide for Care and Use of Laboratory Animals and approved by the MIT and Broad Institute animal care and use committees.

The dataset contains recordings from 16 different individuals in 8 pairs, sampled at $48 \mathrm{kHz} .36$ recording sessions were done, each between 30 and $150 \mathrm{~min}$ in duration. Total duration of all sessions is $38 \mathrm{~h}$. Each recording session yields two mono wav audio files, one for each member of the dyad under study. After each session, the audio files are manually aligned and annotated using Audacity software version 2.1.3. ${ }^{1}$ The data are annotated by researchers for the occurrence of eight different call types: Trill, Twitter, Phee, Chirp, Tsik, Ek, Trillphee, and Chatter. Figure 1 shows an example spectrogram of each of the eight call types.

Annotation is primarily done by inspecting spectrograms. Attribution of calls to either of the two animals wearing a microphone is based on amplitude, reverb, and distinctiveness of the spectrogram image. The calls that can not be attributed are considered to be from other animals in the room and classified as noise. To classify call types, we start by using published classifications (Bezerra and Souto, 2008; Epple, 1968; Watson and Buchanan-Smith, 2018), but some call types are either very uncommon or difficult to distinguish from other call types. For example, we do not distinguish "Loud Shrill," and "Seep" (Watson and Buchanan-Smith, 2018). For training the network, we use the call types for which we have at least 80 exemplars. A total of 15970 marmoset calls labeled by humans are used for training the network.

The audio files are split into $500 \mathrm{~ms}$ segments with $70 \%$ overlap. Table I shows the number of segments for each call type in the dataset, including the noise category. Spectrograms of the segments are generated using a hamming window, size $10.7 \mathrm{~ms}$ and $82 \%$ overlap, yielding spectrogram images of size $257 \times 256$. To each pair of spectrograms, one for each simultaneously recorded channel, we assign a label based on whether there is a human annotation of a call in the middle $150 \mathrm{~ms}$ of the window, including the call type and which animal does the call (ch 1 or ch 2). If no labeled call is present in the segment, the pair is labeled as noise. Since the vocalizations are brief and there is some time in between vocalizations, the data is heavily unbalanced with the vast majority of the labels being noise. To alleviate this, we only include $20 \%$ of the segments labeled as noise into the training and evaluation datasets. Testing uses all the data. We designate three sessions for evaluation and another three full sessions for testing. The training dataset is composed of the remaining 30 sessions. We have several recordings for each pair of individuals and there can be recordings from same individuals in both training and test/evaluation sets.

The basic structure for our network was chosen based on small scale tests and previous knowledge of the authors. In Table III, we show that this structure performs better than 

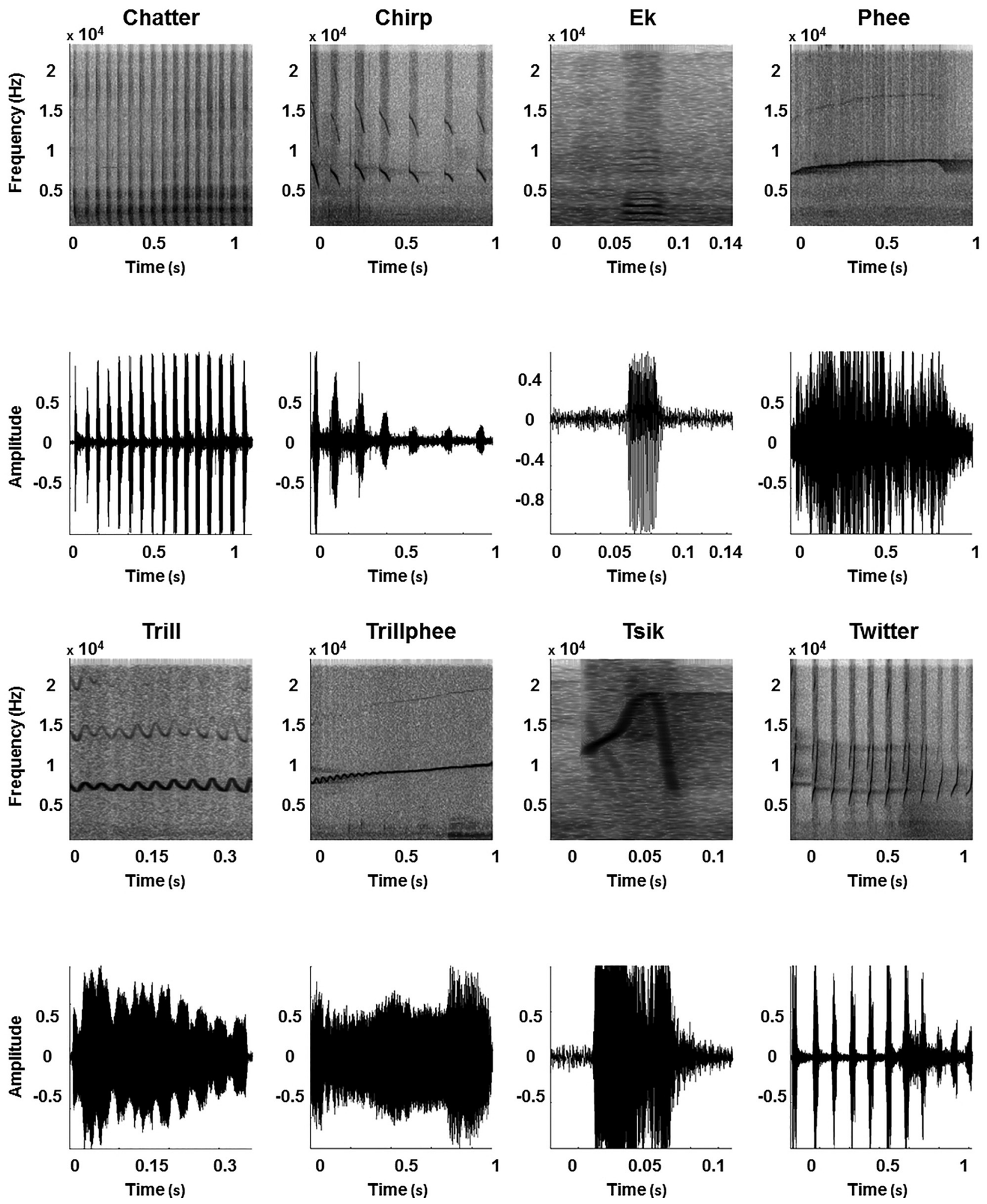

FIG. 1. Examples (spectrograms and wave plot) of call types and noises. Row 1-2 (1-r): Chatter, Chirp, Ek, Phee. Row 3-4 (1-r): Trill, Trillphee, Tsik, Twitter. Row 5-6: four different examples of noise.

variations with different depth or complexity. This structure is an end to end feedforward convolutional neural network with four blocks of two convolutional layers followed by a max pooling layer for each of the two spectrogram images that are fed in simultaneously. The convolutional streams are processed separately, after which the two streams are concatenated and followed by two fully connected layers.
Each layer uses rectified linear units (Nair and Hinton, 2010) as activation functions, except for the final layer which uses the softmax function. Figure 2 shows the architecture of our standard model. Each model we test shares this architecture except for when stated otherwise.

Our models are trained for 74000 iterations with minibatch size of 25 , which takes about $7 \mathrm{~h}$ using a single GPU 


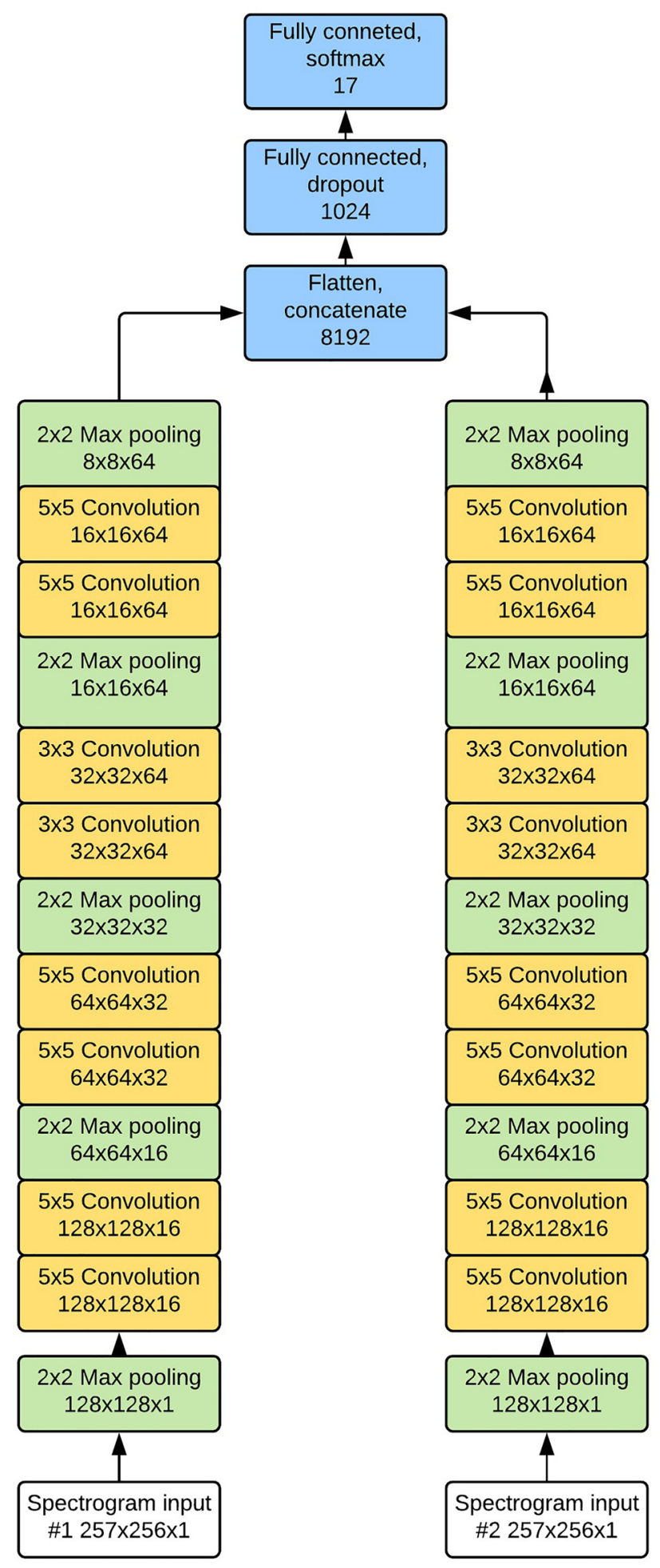

FIG. 2. (Color online) The architecture of the standard dual stream version of our network. The kernel size of each layer is shown before the type of the layer and the dimensions of the layer (excluding batch) are below. Strides of 1 are used for each convolutional layer and strides of 2 for each max pooling layer.

on a computing cluster. The network uses Adam optimizer (Kingma and $\mathrm{Ba}, 2015$ ) and a cross entropy loss function with an exponentially decreasing learning rate that starts at $3 \times 10^{-4}$ and epsilon of $10^{-3}$. Figure 3 shows training and evaluation accuracy during training of the standard model.

Since our network does classification and attribution at the same time, the design of the final layer can be tailored

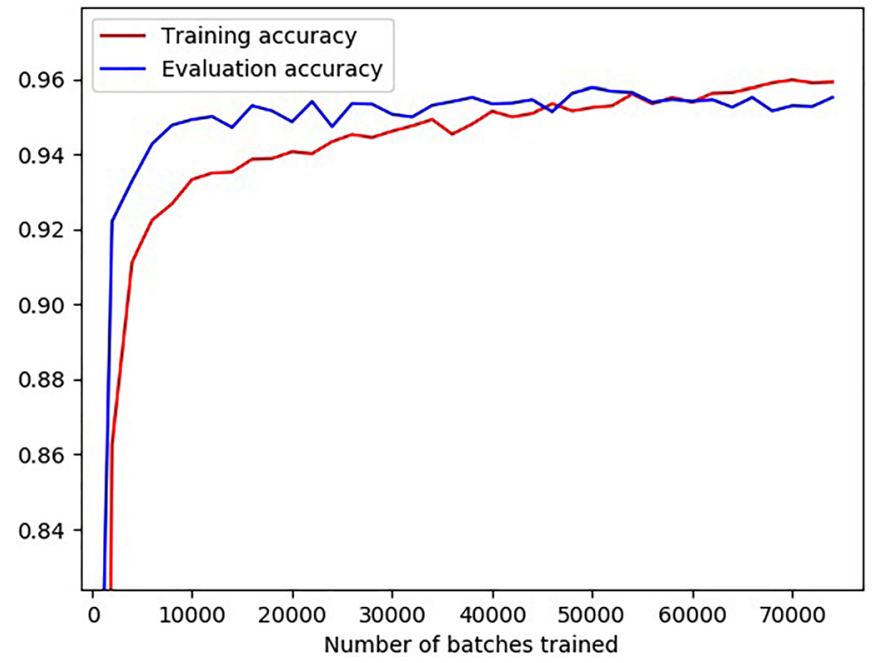

FIG. 3. (Color online) Development of training and evaluation accuracies of the standard network (color online).

accordingly. We experiment with four different layouts for the final layer, shown in Fig. 4. The final layers with 17 and $9 \& 3$ units can only detect a call from one animal at a time. We also test a $9 \& 9$ final layer and a 17 unit multi-class final layer that uses sigmoid activation functions for the final layer instead of softmax, so it can detect several calls at a time (2 stream multi-class in Table III). For predicting with the multi-class network we only look at the highest prediction of the network for each animal since it is not possible for a single animal to make two calls at the same time.

We also test how beneficial it is to use two input streams by comparing our results against those achieved by training a network of the same structure but with only one input stream being fed one of the audio files at a time and only classifying the call made by that animal.

On our experiments we use a small random vertical and horizontal shift (up to 2\%) of the input the array while feeding overflowing values on the other end of the array. We also randomize which input gets fed into input 1 and input 2 and adjust the labels accordingly in order to try and keep the network "speaker" independent instead of learning to recognize each monkey or recording. We explored using more data augmentation methods such as adding random noise and modifying amplitude but those proved to not improve performance and are therefore not used. The standard version of our network does not use batch normalization (Ioffe and Szegedy, 2015).

The goal of our project is to detect, classify, and attribute each call in a pair of long recordings, which includes getting a good temporal accuracy. To evaluate performance, we run the network with $500 \mathrm{~ms}$ window size and $90 \%$ overlap on the test data. To produce each prediction, we take the average of the predictions using a window centered around the $50 \mathrm{~ms}$ we are predicting, and the windows shifted by 50 and $100 \mathrm{~ms}$ to both directions. We then apply a cutoff to this average, such that predictions where the network's probability (value of the output layer) that the element belongs to the class is less than a certain cutoff are classified as noise. This is done for all call types except trillphee, which is a mix between trill and phee: if the highest prediction is trill or phee, 
(A)

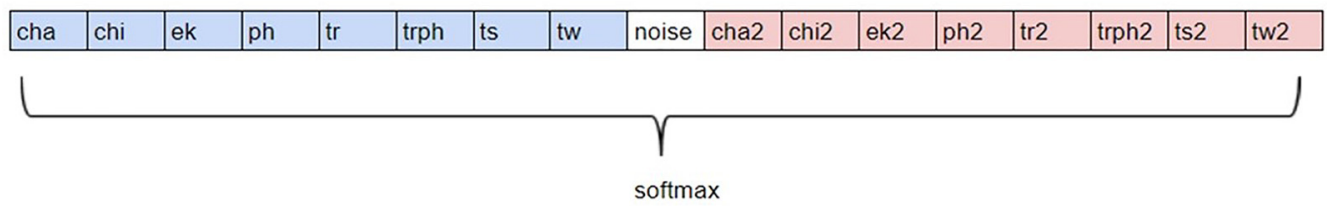

(B)
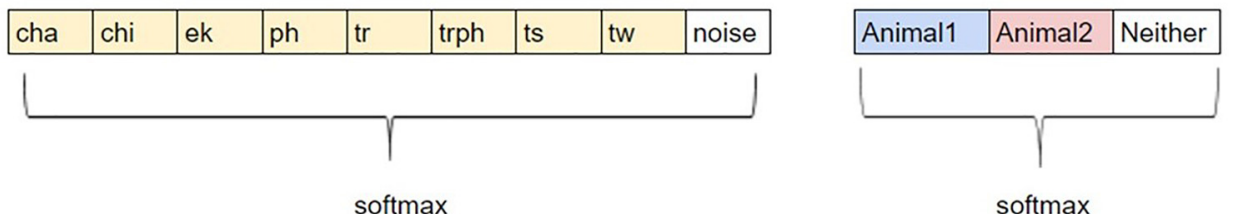

(C)

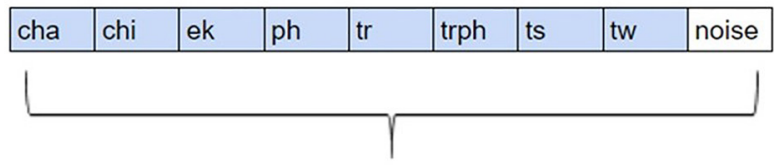

softmax

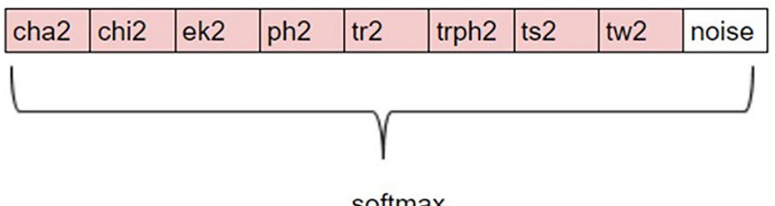

(D)

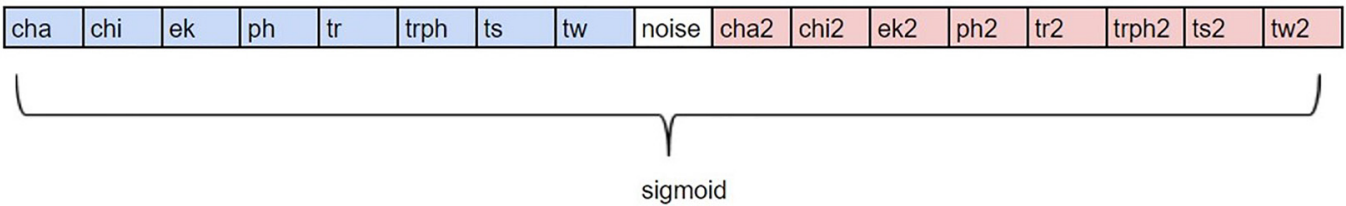

FIG. 4. (Color online) A: The standard 17 unit final layer. B: The $9 \& 3$ final layer setup. If at least one of these layers outputs noise the final output will be noise. C: $9 \& 9$ final layer setup, D: 17 unit multiclass final layer. C and D are capable of detecting two calls at the same time, unlike A and B.

we combine the probability of that and trillphee before applying the cutoff; if highest is trillphee, we sum it together with probabilites for trill and phee before applying cutoff. We found that cutoff values around 0.8 produce the best results.

The network's performance is measured by discretizing the ground truth into $50 \mathrm{~ms}$ segments and labeling each of these according to the original labels. We then test the accuracy of our model on this data. Table II shows the definitions of the metrics we use to evaluate our results. $F 1$-score is the main metric we use when comparing performance of different models.

\section{RESULTS}

We test different versions of our network using cutoff values of $0,0.3,0.4,0.5$, and $0.6-0.95$ with increments of

TABLE II. Definition of terms.

\begin{tabular}{|c|c|}
\hline Term & Explanation \\
\hline True positive (TP) & $\begin{array}{l}\text { There is a call in the frame and it was correctly clas- } \\
\text { sified by the network }\end{array}$ \\
\hline False positive $(\mathrm{FP})$ & $\begin{array}{l}\text { There is no call in the frame, but the network pre- } \\
\text { dicted a call }\end{array}$ \\
\hline True Negative (TN) & $\begin{array}{l}\text { There is no call and the network predicted no } \\
\text { call(=noise })\end{array}$ \\
\hline False Negative (FN) & $\begin{array}{l}\text { There is a call but the network predicted a wrong } \\
\text { call, or no call }\end{array}$ \\
\hline Recall & $\# \mathrm{TP} /(\# \mathrm{TP}+\# \mathrm{FN})$ \\
\hline Precision & \#TP/(\#TP+\#FP) \\
\hline$F 1$-score & $2 *$ Recall*Precision/(Recall+Precision $)$ \\
\hline Accuracy & $\begin{array}{c}\text { Standard framewise accuracy, }(\# \mathrm{TP}+\# \mathrm{TN}) / \\
(\# \mathrm{TP}+\# \mathrm{FP}+\# \mathrm{TN}+\# \mathrm{FN})\end{array}$ \\
\hline
\end{tabular}

0.05 . Figure 5 shows how changing the cutoff affects recall, precision, and $F 1$-score.

Table III shows the best $F 1$-score obtained by each version among the tested cutoffs. As a baseline example we also train a single stream model with the AlexNet (Krizhevsky et al., 2012) architecture on our data.

Our best performing model is able to achieve an F1score of 0.8083 , and a framewise accuracy of 0.9916 on the test set, with the accuracy being significantly higher than $F 1$ score due to vast majority of frames being noise. We can see that small changes to network architecture do not result in very significant differences in final performance, all nine

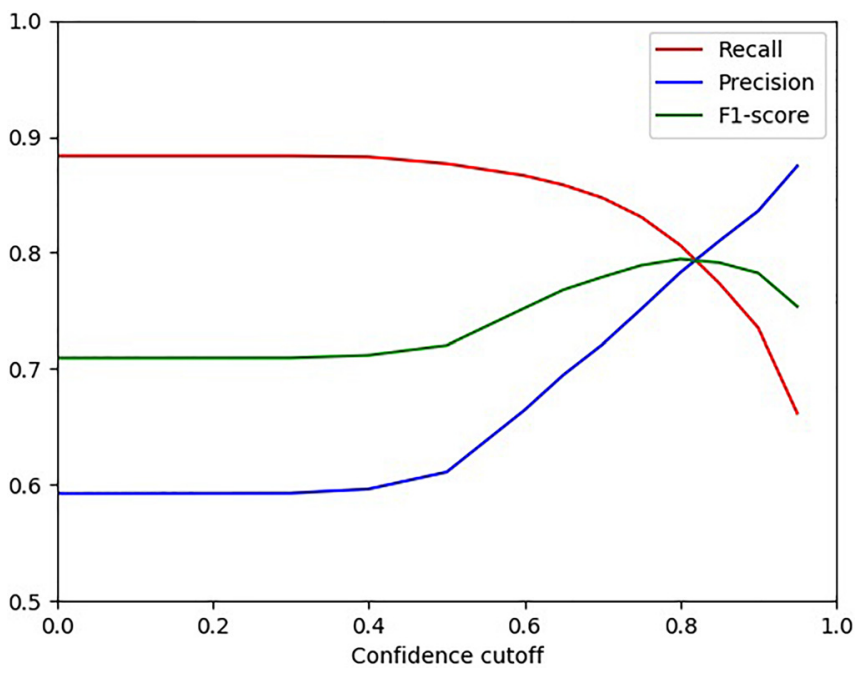

FIG. 5. (Color online) Recall, precision, and $F 1$-score of the standard model (17 output nodes) as a function of cutoff. 
TABLE III. Test accuracy, recall, precision and best $F 1$-scores for all models tested.

\begin{tabular}{|c|c|c|c|c|}
\hline Model & Test accuracy & Recall & Precision & Best $F 1$-score \\
\hline Single stream AlexNet & 0.9887 & 0.7674 & 0.7109 & 0.7381 \\
\hline Single stream standard & 0.9906 & 0.7864 & 0.7674 & 0.7768 \\
\hline Two stream standard (four blocks) & 0.9910 & 0.7925 & 0.7804 & 0.7945 \\
\hline Two stream with twice the amount of filters & 0.9907 & 0.8181 & 0.7561 & 0.7793 \\
\hline Two stream, five blocks & 0.9905 & 0.8026 & 0.7573 & 0.7793 \\
\hline Two stream, three blocks & 0.9910 & 0.8184 & 0.7672 & 0.7920 \\
\hline Two stream standard with $9 \& 3$ output layers & 0.9913 & 0.8170 & 0.7770 & 0.7965 \\
\hline Two stream with $9 \& 9$ output layers & 0.9916 & 0.8468 & 0.7731 & 0.8083 \\
\hline Two stream with $9 \& 9$ output layers and batch normalization & 0.9914 & 0.8262 & 0.7758 & 0.8002 \\
\hline Two stream multi-class (17 output) & 0.9918 & 0.8123 & 0.7961 & 0.8042 \\
\hline Human researcher & 0.9889 & 0.8508 & 0.6896 & 0.7618 \\
\hline
\end{tabular}

networks utilizing dual audio input reach $F 1$-scores between 0.7793 and 0.8083 . Interestingly, batch normalization (two stream with 9\&9 output layers and batch norm in Table III) does not improve the performance of our network and instead seems to result in slightly lower accuracies. This might be because batch normalization has a regularizing effect, and/or network is already using dropout, so the combination of both might be reducing the expressive power of the network too much. The layout of the final layer does not seem to noticeably affect network performance, with the exception that networks capable of detecting two simultaneous calls seem to perform slightly better than ones that can not, with all of those reaching $F 1$-scores over 0.8 , while none of the ones that can only detect a single call at a time do. Figure 6 shows an example of the network detecting two different calls at the same time. Training the network with categories evened out produces a high false positive rate with an $F 1$-score of 0.1633 .
Our results show that using two audio streams improves performance. Each network that uses two input channels beats every network that uses only one input. The margin is not very wide, the best performing single stream reaches an $F 1$-score only 0.0025 lower than the worst performing two stream network's score. This difference is most likely smaller because the networks with single input can detect two simultaneous calls while the worst performing two stream networks can not. Regardless, the difference is clear enough to see that two inputs are beneficial.

Our network is trained to replicate the labels of human observers, yet between human observers, there is inherent variability. Our database is annotated by only a single observer per observation. To better appraise the performance of the network, it is useful to know whether the difference between the network and the human observer is bigger or smaller than the difference between human observers. Therefore, we ask a different human observer to re-label the

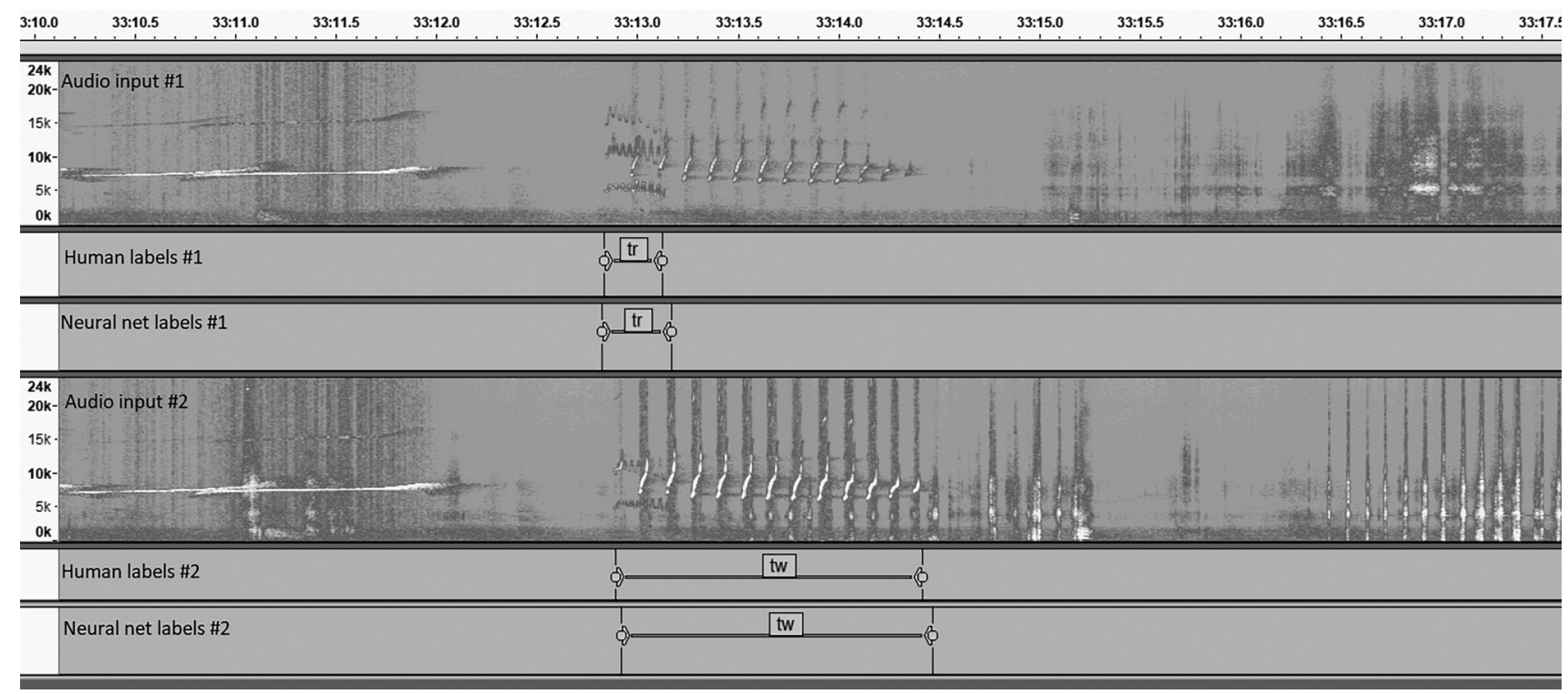

FIG. 6. An example of our best performing model detecting two different calls simultaneously on a test session. The recording is from two marmosets housed in the same cage, each wearing a microphone located at the chest (as all the recordings in our dataset). The top spectrogram shows animal 1 , and the bottom spectrogram shows animal 2. Many marmoset calls are visible in both spectrograms, yet certain marmoset calls are clearly more pronounced on one channel than on the other. Between time points 33.10 and 33.12 there is a "phee" call which is equally pronounced on both mics, and likely was produced by an animal in another cage. At 33.13, a "trill" call occurs. It is most pronounced on ch1, and this was labeled as a call coming from animal 1, but both human observer and the network. At the same time, a "twitter" call begins. This is more pronounced on ch 2 and is labeled as such by both human and network. This figure was created using Audacity software (footnote 1). 
TABLE IV. $F 1$-scores for human relabels and our best model.

\begin{tabular}{lcccc}
\hline \hline Ground truth & Original & Original & Human relabel & Human relabel \\
\hline Predictions & Computer & Human relabel & Computer & Original \\
Best $\boldsymbol{F}$ 1-score & 0.8083 & 0.7618 & 0.7858 & 0.6694 \\
\hline \hline
\end{tabular}

test sessions and test how closely these labels match the original observer using the same metrics we used for measuring the performance of our network. The result shows that the second human observer is less accurate than our network in replicating the original labels ("human researcher," Table III). This underscores not only that there is variability between observers, but also that our network can perform better than some human observers given this set of training data.

Inspired by these results, we also measure accuracy again using the labels created by the second human observer (human relabel) as ground truth instead of the original ones. The results of this experiment are shown in Table IV. Our best network achieves an $F 1$-score of 0.7858 when compared to the human relabels, while the original human labels only reaches an $F 1$-score of 0.6694 in this comparison. Our networks' predictions are closer to both human labels than they are to each other.

We also test our network on the marmoset call dataset shared introduced in Turesson et al. (2016). This dataset consists of 321 segmented calls, so it is much smaller than our dataset, and because the calls are pre-segmented, the task is one of classification, not detection. Our network is optimized for larger datasets, and as such, has the risk of overfitting to smaller dataset; however, we are able to achieve good results. We train a single stream normalized version of our network from scratch for 600 batches of 25 examples 10 times while using $90 \%$ of the calls in each call type for training, and evaluate accuracy on the remaining $10 \%$ (randomly selected). For training, we create spectrogram images of $500 \mathrm{~ms}$ sliding window with $200 \mathrm{~ms}$ steps over the duration of each call and label each image with the label of the call. For prediction, we run the network with each window of the call and use the mean of those as our prediction. Table $\mathrm{V}$ shows our results measured by the metrics defined by Turesson et al. We find out that our network's accuracy is better than the best model tested by Turesson et al., which is a Support Vector Machine (SVM) model with Linear Predictive Coding (LPC).

We implement our network into an easy to use program that can classify marmoset recordings. We also optimize its performance, which allows our network to classify (including spectrogram transformations) an hour long audio recording in 8 min using a laptop with Nvidia GeForce GTX 1060 graphics card and Intel Core i5 processor. Our model as well

TABLE V. Performance of our network on the Turesson et al. (2016) dataset and comparison with their model.

\begin{tabular}{lcc}
\hline \hline Model & $F 1$-score & Accuracy \\
\hline Our network trained from scratch & 0.890 & 0.936 \\
SVM with LPC (their best) & 0.852 & 0.843 \\
\hline \hline
\end{tabular}

as source code used for the results in this paper and a subset of our data are freely available at http://marmosetbehavior. mit.edu (Oikarinen et al., 2019).

\section{DISCUSSION}

The end-to-end feedforward Convolutional Neural Network (CNN) introduced in this article is one of a kind, in that it is capable of automatic call detection, classification of call types, and attribution of the caller, all together. The network automatically detects whether any given segment of hour long audio files of dual channel marmoset vocal recordings contains a call or not. It then classifies the detected calls into one of nine types (eight call types + one noise category), and finally attributes the identity of the call to the animals wearing the microphone (either of the two animals or neither). In addition, we have adapted the CNN for single stream audio recordings as well. To our knowledge, this task optimized feedforward CNN gives the best performance to date on automatic vocal classification of marmoset calls.

Unlike previous efforts that classify call types on noisefree, pre-segmented audio (Agamaite et al., 2015; Turesson et al., 2016), our network uses raw, noisy spectrograms. The network is thus robust in detecting and classifying in an environment that is noisy, and does not require any preprocessing of the input audio stream. Agamaite et al. (2015) describe a quantification of audio features, chosen by the investigators, to identify different call types. Here, we let the network learn the useful features that it needs to enable the trio of detection, classification, and caller attribution.

Our approach is image-based, using the spectrogram of the audio. However, our dataset is different from many other image datasets. Typically, large benchmark datasets like ImageNet (with $15 \times 10^{6}$ images, and thousands of categories) used in testing and validating machine learning algorithms and neural networks are well curated. New object recognition algorithms typically use a uniform number of each of the different categories to train their networks. Unlike such datasets, our training set is quite modest in size and is not curated (noise-free, anti-aliased, mean-centered, etc.,), and not trained with a uniform number of samples of different calls (e.g., more "trill," or "phee," than "chatter") because not all calls are equally common, yet all calls are potentially important to study. Regarding the sliding window of analysis, the size of the window may affect performance. There is a tradeoff between long windows being better for detecting long vocalizations and short windows being better for detecting short duration signals. Small scale preliminary tests lead us to choose a length of $500 \mathrm{~ms}$ as the optimal length. Future efforts might benefit from using multiple- or adaptive window lengths.

Turesson et al. (2016) used a very small, but well curated dataset (321 calls) which they have made freely available. They applied several machine learning and neural network algorithms and found that an SVM with LPC had the best performance metrics. Training our network from scratch achieved better than their top performance. This shows that our convolutional network contains robust learned representations and that it can classify marmoset 
calls recorded in different environmental scenarios. We believe even better results could be achieved with this dataset using our model after careful hyperparameter optimization. Besides other marmoset datasets, it is very well possible that the convolutional network we have designed here is capable of transfer learning to be adapted for vocal classification in other species. Our shared code is freely available for others to use.

Our study diverges from Zhang et al. (2018) in that we recorded from two animals instead of one which is a step towards analyses on social behavior but required us to solve the problem of source attribution. Similar to Agamaite et al. (2015), Zhang et al. (2018) hand-picked three short-time acoustic features that are extracted for each audio frame: energy, peak-ratio of autocorrelation (PRA), and log melfilter bank spectrum. It is these features that are used for detecting voiced vs non-voiced segments, and later to train the network on the extracted log mel-filter bank spectra. The call detection employed was a rule-based threshold detection algorithm that is applied in many human speech processing systems for voice activity detection (VAD). Thus, call detection and classification were done separately, with a rulebased approach for detection followed by a neural network training for call classification. In our approach, we directly feed our network raw, noisy spectrograms from the dual channels that contain background animal calls, and a variety of noise in the environment. Detection, classification, and attribution are performed by a single network and we avoid the possible bias that could be introduced by hand-picking features. Our effort with separate detection and classification models (Sharma et al., 2017) achieved 80.5 and $88.25 \%$ accuracy for detection and classification, respectively, and therefore lower than the performance of the network we present here. The neural network of Zhang et al. (2018) is a fully connected recurrent neural network (RNN) with LSTM, while ours is a feedforward deep convolutional neural network. We found that adding LSTM layers to our system does not improve the performance on the task.

Many systems have applied the architecture of AlexNet (Krizhevsky et al., 2012) to a high degree of success. In that vein, we trained an AlexNet model on a single stream of our dataset with the raw spectrograms as the input images. The performance of this network was much worse than our convolutional network (both single and dual stream audio). Newer models are available, such as ResNet (He et al., 2015). However, ResNet is much bigger than our model or AlexNet, and would be slower and have higher hardware requirements to run. Also, our task is smaller than ImageNet in terms of number of classes and number of examples. The performance of our network for the data set from Turesson et al. (2016) shows that as a proof of principle, we have a task optimized convolutional network that has learned features generalized over the space of vocal calls.

We show that our network more closely replicates labelling from a human observer than a second human observer is able to replicate the first human observer. This highlights that there is considerable variation in human labelling, even among experts. In the existing literature, there is considerable variation in the definition and number of call types distinguished (e.g., Bezerra and Souto, 2008; Epple, 1968; Watson and Buchanan-Smith, 2018). Further improvement of auto-detection and classification efforts will be aided by standardization of the definition of call types (e.g., "phee," "short phee," "long phee," etc.), naming conventions, and a robust, yet flexible dataset (with noise, multiple streams, etc.). Additional areas of improvements include having an expanded training set, labels, and timings of the calls corroborated by multiple humans to increase reliability.

\section{ACKNOWLEDGMENTS}

The authors wish to thank the following for their generous support: The Stanley Center for Psychiatric Research, the Poitras Center for Psychiatric Disorders Research, the Simons Center for the Social Brain, the TanYang Center for Autism Research, and NIH (Award No. 1R01MH111916-01A1).

\footnotetext{
${ }^{1}$ Audacity ${ }^{\circledR}$ software is copyright $1999-2018$ Audacity Team. The name Audacity ${ }^{\circledR}$ is a registered trademark of Dominic Mazzoni.
}

Agamaite, J. A., Chang, C.-J., Osmanski, M. S., and Wang, X. (2015). “A quantitative acoustic analysis of the vocal repertoire of the common marmoset (Callithrix jacchus)," J. Acoust. Soc. Am. 138(5), 2906-2928.

Bezerra, B. M., and Souto, A. (2008). "Structure and Usage of the vocal repertoire of Callithrix jacchus,” Int. J. Primatol. 29(3), 671-701.

Boddapati, V., Petef, A., Rasmusson, J., and Lundberg, L. (2017). "Classifying environmental sounds using image recognition networks," Proc. Comput. Sci. 112, 2048-2056.

Eliades, S. J., and Miller, C. T. (2017). "Marmoset vocal communication: Behavior and neurobiology,” Dev. Neurobiol. 77(3), 286-299.

Epple, G. (1968). "Comparative studies on vocalization in marmoset monkeys (Hapalidae)," Folia Primatologica 8(1), 1-40.

Fuller, J. L. (2014). "The vocal repertoire of adult male blue monkeys (Cercopithecus mitis stulmanni): A quantitative analysis of acoustic structure," Am. J. Primatol. 76(3), 203-216.

Giret, N., Roy, P., Albert, A., Pachet, F., Kreutzer, M., and Bovet, D. (2011). "Finding good acoustic features for parrot vocalizations: The feature generation approach," J. Acoust. Soc. Am. 129(2), 1089-1099.

Graves, A., Mohamed, A., and Hinton, G. (2013). "Speech recognition with deep recurrent neural networks," arXiv:1303.5778.

He, D., Zhang, X., Ren, S., and Sun, J. (2015). "Deep residual learning for image recognition," arXiv:1512.03385.

Hedwig, D., Hammerschmidt, K., Mundry, R., Robbins, M. M., and Boesch, C. (2014). "Acoustic structure and variation in mountain and western gorilla close calls: A syntactic approach,” Behaviour 151, 1091-1120.

Henry, L., Craig, A. J. F. K., Lemasson, A., and Hausberger, M. (2015). "Corrigendum: Social coordination in animal vocal interactions. Is there any evidence of turn-taking? The starling as an animal model," Front. Psychol. 6, 1924.

Ioffe, S., and Szegedy, C. (2015). "Batch normalization: Accelerating deep network training by reducing internal covariate shift," in Proceedings of the 32nd International Conference on International Conference on Machine Learning - Volume 37 (ICML'15), July 6-11, Lille, France, pp. 448-456.

Jennings, C. G., Landman, R., Zhou, Y., Sharma, J., Hyman, J., Movshon, J. A., Qiu, Z., Roberts, A. C., Roe, A. W., Wang, X., Zhou, H., Wang, L., Zhang, F., Desimone, R., and Feng, G. (2016). "Opportunities and challenges in modeling human brain disorders in transgenic primates," Nat. Neurosci. 19(9), 1123-1130.

Kingma, D. P., and Ba, J. L. (2015). "Adam: A method for stochastic optimization," in Proceedings of the International Conference on Learning Representations 2015, May 7-9, San Diego, CA.

Kobayasi, K. I., and Riquimaroux, H. (2012). "Classification of vocalizations in the Mongolian gerbil, Meriones unguiculatus," J. Acoust. Soc. Am. 131(2), 1622-1631. 
Krizhevsky, A., Sutskever, I., and Hinton, G. E. (2012). "ImageNet classification with deep convolutional neural networks," Adv. Neural Inf. Process. Syst. 25, 1097-1105.

Levinson, S. C., and Torreira, F. (2015). "Timing in turn-taking and its implications for processing models of language," Front. Psychol. 6, 731.

Miller, C. T., Freiwald, W. A., Leopold, D. A., Mitchell, J. F., Silva, A. C., and Wang, X. (2016). "Marmosets: A neuroscientific model of human social behavior," Neuron 90(2), 219-233.

Miller, C. T., Mandel, K., and Wang, X. (2010). "The communicative content of the common marmoset phee call during antiphonal calling," Am. J. Primatol. 72(11), 974-980.

Nair, V., and Hinton, G. E. (2010). "Rectified linear units improve restricted Boltzmann machines," in Proceedings of the 27th International Conference on International Conference on Machine Learning (ICML'10), June 21-24, Haifa, Israel, pp. 807-814.

Oikarinen, T., Srinivasan, K., Landman, R., Meisner, O., Hyman, J. B., Parmar, S., Fanucci-Kiss, A., Desimone, R., Landman, R., and Feng, G. (2019). "Marmoset behavior," http://marmosetbehavior.mit.edu/ (Last viewed January 21, 2019).

Pettitt, B. A., Bourne, G. R., and Bee, M. A. (2012). "Quantitative acoustic analysis of the vocal repertoire of the golden rocket frog (Anomaloglossus beebei)," J. Acoust. Soc. Am. 131(6), 4811-4820.
Prat, Y., Taub, M., and Yovel, Y. (2016). "Everyday bat vocalizations contain information about emitter, addressee, context, and behavior," Sci. Rep. 6(1), 39419.

Sacks, H., Schegloff, E. A., and Jefferson, G. (1974). "A simplest systematics for the organization of turn-taking for conversation," Language 50(4), 696-735.

Sharma, S., Landman, R., Srinivasan, K., Cheung, R. T., Sharma, J., Sur, M., Feng, G., and Desimone, R. (2017). "Using machine learning for automated animal call detection and classification," Program No. 530.05, 2017 Neuroscience Meeting Planner, Society for Neuroscience, Washington, DC.

Soltis, J., Alligood, C. A., Blowers, T. E., and Savage, A. (2012). "The vocal repertoire of the Key Largo woodrat (Neotoma floridana smalli)," J. Acoust. Soc. Am. 132(5), 3550-3558.

Turesson, H. K., Ribeiro, S., Pereira, D. R., Papa, J. P., and De Albuquerque, V. H. C. (2016). "Machine learning algorithms for automatic classification of marmoset vocalizations," PLoS ONE 11(9), e0163041.

Watson, C. F. I., and Buchanan-Smith, H. M. (2018). "Common marmoset care," http://www.marmosetcare.com/ (Last viewed January 1, 2018).

Zhang, Y.-J., Huang, J.-F., Gong, N., Ling, Z.-H., and Hu, Y. (2018). "Automatic detection and classification of marmoset vocalizations using deep and recurrent neural networks," J. Acoust. Soc. Am. 144(1), $478-487$. 